\title{
Trauma-related impairment in children-A survey in Sri Lankan provinces affected by armed conflict ${ }^{\frac{1}{4}}$
}

\author{
Thomas Elbert ${ }^{\mathrm{a}, \mathrm{b}, *}$, Maggie Schauer $^{\mathrm{a}, \mathrm{b}}$, Elisabeth Schauer ${ }^{\mathrm{b}}$, Bianca Huschka ${ }^{\mathrm{b}}$, \\ Michael Hirth ${ }^{c}$, Frank Neuner ${ }^{b, d}$ \\ a University of Konstanz, Department of Psychology, Feuersteinstr. 55 , \\ Haus 22, D-78479 Reichenau-Lindenbühl, Germany \\ b vivo, Germany \\ c German Technical Cooperation (GTZ), Germany \\ 'Bielefeld University, Germany
}

\section{Keywords:}

Post-traumatic stress disorder

Organised violence

School performance

Memory

Building block effect

Dose effect

Prevalence

\section{A B S T R A C T}

Objectives: The present study examined traumatic experiences, PTSD, and co-morbid symptoms in relation to neuropsychological and school performance in school children affected by two decades of civil war and unrest.

Method: The epidemiological survey of children's mental health included a representative sample of 420 school children. Local teachers were trained to administer a translation of the UCLA-PTSD Index Form. The instrument and the epidemiological findings were validated by assessment through clinical expert interview, school grades, and neuropsychological testing in a subsample $(N=67)$.

Results: Ninety-two percent of the children surveyed had experienced severely traumatizing events such as combat, bombing, shelling, or witnessing the death of a loved one. Twenty-five percent met the criteria for a diagnosis of PTSD. Traumatized children reported lasting interference of experiences with their daily life, which was corroborated by memory testing, scores in school performance and ratings of social withdrawal. Depressive symptoms and poor physical health were frequent in these children. The majority of trained teachers achieved valid results in the structured interviews.

Conclusion: Performance and functioning in children are related to the total load of traumatic events experienced. An important component of psychosocial programs in postconflict areas should include increasing community-based awareness of the consequences of traumatic stress, both as a preventative measure and as a way of decreasing stigmatization of affected individuals.

\section{Introduction}

Traumatic experiences have been common in Sri Lanka's North-Eastern provinces. For two decades, conflict and violence have killed people by the tens of thousands and displaced hundreds of thousands more in Sri Lanka. The LTTE (the Liberation Tigers of Tamil Eelam) has been fighting for autonomy, resulting in areas of permanent conflict in the North and East of the country. Civilians have become casualties of war; landmines and unexploded ordnance pose a constant threat; hospitals and

\footnotetext{
Work was supported by GTZ, DRF and vivo.

* Corresponding author at: University of Konstanz, Department of Psychology, Feuersteinstr. 55, Haus 22, D-78479 Reichenau-Lindenbühl, Germany.
} 
schools have been destroyed, the economy has been shattered. Even in the most remote of places, not a single school can be seen that has escaped serious damage due to bombing and shelling. At the sight or sound of airplanes, children become severely frightened and quickly hide. The conflict has resulted in periodic mass displacement of the population. A cease-fire has been in place since December 2001 but little progress has been made in peace negotiations between the government and the LTTE and as of December 2005 violence has escalated again.

Children are a particularly vulnerable group that suffers from repeated traumatic war experiences, loss of beloved ones, displacement, lack of food, poverty, domestic violence and interruption of school. This research has looked to quantify the impact and correlation of these factors by means of a survey in the regions, which are most affected by the civil war. In particular, the magnitude and type of psychological and social problems that affect traumatized children were assessed. Mental health problems resulting from traumatic experiences, including PTSD, depression, and fear-related cognitive and emotional impairment, are known as potent barriers that deny children of their rights to lead a loving relationship with family and friends and to perform adequately in school. According to the United Nations Convention on the Rights of the Child (UNCRC), which has been ratified by 191 countries. States have an obligation to ensure that has much as possible every child's survival and development. A number of investigations from various war zones in different cultures have reported high rates of PTSD in children exposed to traumatic events. Saigh (1991) reported that $20 \%$ of Lebanese children who had been exposed to bombings and terror attacks met the criteria for PTSD. Post-traumatic stress reactions have been observed in 41\% of Palestinian children from Gaza (Thabet \& Vostanis, 2000), in refugee children from Cambodia (Kinzie, Sack; Angell, Clarke, \& Ben, 1989; Realmuto et al., 1991), and Central America (Arroyo \& Eth, 1985), with up to 50\% of children meeting criteria of PTSD some 5 years after their exposure to war atrocities. Smith, Perrin, Yule, Hacam, and Stuvland (2002) collected data from a community sample of 2976 war-affected Bosinan children, aged between 9 and 14 years. Half the children presented with PTSD symptoms without significant differences between age groups. Similarly, after the genocide in Rwanda, more than half of the Rwandese children were at risk for developing PTSD (Dyregrov, Gupta, Gjestad, \& Mukanoheli, 2000). In orphans, Schaal and Elbert (2006) found that PTSD persisted at a rate of $44 \%$ even more than 10 years after the genocide. Post traumatic stress reactions of children in Rwanda were associated with parental loss, violence exposure, and most importantly, the feeling that their life was in danger (Dyregrov et al., 2000; Schaal \& Elbert, 2006).

In children as well as in adults, cumulative exposure to war trauma constitutes a risk factor for lasting, that is, chronic mental health problems (Neuner et al., 2004a; Schaal \& Elbert, 2006; Smith et al., 2002; Thabet \& Vostanis, 2000). Even few severe traumatic experiences may lead to mental health problems in children. Repeated exposure to various traumatic events will however lead to a near linear increase in the proportion of survivors who are traumatized, until ultimately all have been affected (Neuner et al., 2004a; Schaal \& Elbert, 2006). Somasundaram (2002) describes how children in Sri Lanka have been "traumatised by common experiences such as shelling, helicopter strafing, round ups, cordon and search operations, deaths, injury, destruction, mass arrests, detention, shootings, grenade explosions and landmines" (p. 1269) and presents a brilliant qualitative outline, which we see perfectly fits and complements the quantitative findings of the present survey.

Even so individual resilience is variable and dependent upon environmental and genetic (DeQuervain et al., 2007) factors, with a significant and large enough adverse event load, few if any will be protected from developing symptoms of posttraumatic stress. Experiences, such as domestic violence, or living in a war zone, prior to a natural disaster all add to the 'building block effect' (Neuner et al., 2004a) and thus explain variance of PTSD rates in different populations (Neuner, Schauer, Catani, Ruf, \& Elbert, 2006). Children are particularly susceptible when they encounter stressful experiences during sensitive periods of their emotional, cognitive, and endocrinological development (Charmandari, Kino, Souvatzoglou, \& Chrousos, 2003). The age when traumatic stressors had occurred might therefore produce expectable variations in studies with children. In addition, the use of inadequate or not valid instruments may add to disparate findings. In this study, we therefore validated the assessment procedure and quantified the load of traumatic events as the number of different event types experienced.

Often investigations of children's post-traumatic stress reactions have been limited to data that rely solely on parental report. However, Yule and Canterbury (1994) demonstrated disparity between the assessments based on parents and children, and Almqvist and Brandell-Forsberg (1997) found evidence that parents consistently underestimate PTSD symptoms in their children. In the present investigation, we therefore included ratings by the children and their parents as well as validations of the outcome by means of expert interview with the children.

In addition to PTSD, we also evaluated the consequences of traumatic experiences on school performance, as measured by school grades. Given that evidence is emerging that stress generally impairs hippocampal-dependent memory tasks in both humans and rats (e.g. Kim \& Diamond, 2002; McEwen, 2002; Sapolsky, 1992), we also wanted to see if the performance of children in memory tasks varied with traumatic experiences. Learning and memory that rely on hippocampal functioning are susceptible to disruption by stress, mediated in part by the activation of type II corticosteroid (glucocorticoid) receptors (Kim \& Diamond, 2002) and in part by the decoupling of fear structures from autobiographic memory through Hebbian mechanisms (Elbert, Rockstroh, Kolassa, Schauer, \& Neuner, 2006). Inadequate coupling of autobiographic with implicit (emotional and sensory) memory is at the core of PTSD; PTSD-related problems build-up with increasing amount of traumatic stress perceived (Neuner et al., 2004a). Based on this knowledge, we predicted that in children with PTSD, memory performance and school grades (in areas where memory plays an important role) would be weakened in relation to the increasing number of traumatic incidences experienced by the children, while the number of somatic symptoms would go up. 


\section{Methods}

Sample

This survey was designed as a randomized, epidemiological screening of school attending children. Fifth grade children from 16 different primary schools randomly chosen from all 5 administrative zones of the North Eastern Tamil Provinces of Sri Lanka were selected as being representative for this survey. In alphabetical order, every other (or in larger classes every third) child was invited to participate. Informed consent was obtained from both the child and parent or guardian. The study was approved by the Ministry of Education, Sri Lanka, the University of Konstanz Ethical Review board, and by the local assembly of school principals. Guardians of children who were chosen to participate in the interview were asked to complete a parent questionnaire. General acceptance rates were excellent, with $99 \%$ or above participating across schools.

\section{Sample characteristics}

A total of 218 Tamil girls and 202 boys and 398 caregivers responded to the interview questions asked by 23 different teachers. Sixty-six percent of the respondents for the corresponding section of the parent version of the questionnaire were mothers; $27 \%$ were fathers. Eleven percent of the children had lost their fathers and $3 \%$ their mothers.

Eight-five percent of the children were Hindu and 15\% Christian. The ages ranged from 10 to 14 years (average 10.5 years) with $69 \%$ between 10 and 11 years old. The body mass index (body weight in $\mathrm{kg} / \mathrm{squared}$ height in $\mathrm{m}$ ) was $14.5 \pm 1.5$ in boys and girls with a range from 10 to 21 . The number of people living in a household with the child ranged from 0 to 16 (median household size 6). (Certain observations, such as a significantly smaller household size when children had reported that a parent has died, added face validity to these figures).

Only $23 \%$ of the children had never been displaced. Thirty-four percent had been displaced once, $29 \%$ twice and $14 \%$ three to seven times. Figures for the number of years spent in displacement camps varied between schools (Kruskal-Wallis Test, $P<.0001$ ), ranging from a median of 5 years in Jayapuram to no reports of displacement in 4 of the 16 schools. Similarly, the median for last displacement was 7 years ago for Jayapuram, whereas most displacements occurred only shortly before the assessment in Thannirootu (Kruskal-Wallis Test, $P<.0001$ ).

\section{Procedures}

Teachers of the selected classes participated in a five-day psycho-social awareness training with a special focus on how to recognize trauma-affected children. In a second training (1.5 days), the essentials of trauma-spectrum symptoms were taught and instructions were given on how to conduct the survey. In a parent-teacher meeting, often held together with the school principal, parents and caregivers were informed of the goal of the study and on how to complete the parent-version of the questionnaire. More than $80 \%$ of the parents attended these meetings.

The trained teachers administered the questionnaires through individual interviews to the selected sample of children and to one respective primary caregiver of each child, whereby child and parent were interviewed by different teachers. Most parents and caregivers completed the questionnaire during the parent-teacher meetings. The remaining parents were visited by the teachers on an individual basis and asked to participate. Prior to interviews, teachers obtained informed consent from the primary caregiver and the child.

\section{Materials}

Tamil language versions of all measures were obtained through translation and blind back translation (Bracken \& Barona, 1991). A professional counsellor translated from English to Tamil, and independent translators performed the back translation, blind to the original version. We checked discrepancies and a final version were derived through consultation with a bilingual child counsellor.

The following instruments were used in the survey:

Event list. A previously used event list (Karunakara et al., 2004; Neuner et al., 2004a) was modified to fit the conditions in Sri Lanka (event checklists available at www.vivo.org; publications). In order to detect problems of performance and functioning in relation to the load of traumatic experiences, we categorised the traumatic event load as number of different types of traumatic stressors, checked in the list.

\section{Standard of living list}

This measure was specifically adapted to the cultural situation in Northeast Sri Lanka, based on previous experiences by the NGO vivo, in resource-poor countries, and by Sri Lankan surveys (Elbert, Huschka, Schauer, \& Schauer, 2003, available from the authors upon request). 
Assessment of trauma, PTSD and grief. For the assessment of PTSD and grief, both the Child Version and the Parent Version of the UCLA PTSD INDEX FOR DSM IV (Revision 1) were used (Steinberg, Brymer, Decker, \& Pynoos, 2004). Researchers and clinicians at the UCLA Trauma Psychiatry Service have developed this series of self-report instruments to be used to screen for both exposure to traumatic events and for all DSM-IV PTSD symptoms in school-age children and adolescents who report traumatic experiences. These instruments are meant to serve as brief self-report screening tools to provide information regarding trauma exposure and PTSD symptoms. The items of the UCLA PTSD indices are keyed to DSM-IV criteria and can provide preliminary PTSD diagnostic information. However, these instruments are not intended to be used in place of a structured clinical interview to establish a definitive PTSD diagnosis. Instead, the instruments are meant to screen quickly and efficiently for PTSD symptoms in children and adolescents who have experienced a traumatic event, and to provide information regarding the frequency of those symptoms. Consequently, the outcome needed to be validated by expert clinical interviews, which was done by four of the authors of this paper.

\section{Validation of the translated measures and neuropsychological testing}

A random sub-sample of $N=67$ children, chosen from seven randomly selected schools, were interviewed 6-8 weeks later by clinical experts using the help of local counsellors trained as interpreters (English-Tamil). The four interviewers (with degrees from Yale, Konstanz or Tübingen University) were experienced in work with traumatized individuals in different cultural settings. The structured clinical interview followed the CIDI (Composite International Diagnostic Interview) K-section, with some of the questions reformulated in a child-friendly manner following the M.I.N.I. Kid 2002 version 2.0 (Sheehan et al., 1998). In addition, the children were examined following the remainder of the M.I.N.I. Kid version and detailed diagnostic interviews were performed when indicated by the screening questions of this instrument.

In addition, two neuropsychological tests served to examine memory performance in these children; the Rey-Osterrieth Complex Figure test and a test for the memory of places of objects (MP-test). The Rey-Figure assesses visuospatial constructional ability and visual memory. It permits the evaluation of several cognitive processes, including planning and memory function. Results were scored according to Lezak (1995) and analysed using an ANOVA with the factors group (PTSD vs. no PTSD) and time (copy, memory I and memory II). In the MP test each of 10 small familiar objects (a stone, a leaf, a balloon, a shell, a stick, a toffee, etc.) is hidden under a cup. The objects are shown to the child one by one by lifting the cups briefly in sequential order. The child is asked to name each object. Then 10 identical objects and the three so-called distracter objects are placed in front of the child and the child is asked to put each of these on top of the cup where the respective duplicate would be found. The number of correctly assigned objects is counted.

School performance was estimated by academic grades, and in addition, teachers rated the academic skill levels of those children who were participating in the validation study.

Procedure of the validation study. After obtaining informed consent, children were asked to copy the Rey-Figure. The figures were put away and the child was asked to immediately draw the figure from memory. In each case, children were given positive feedback irrespective of the actual performance. After the Rey-Figure test the child was screened for potential PTSD by using the CIDI to conduct the structured interviews. Next, the MP-test was introduced as a game. Again children were reinforced irrespective of their performance. After a short break with a refreshing drink, the M.I.N.I. Kid interview followed. After the interviews were finished, the child was asked to produce a third drawing of the Rey-Figure once again from memory. The whole procedure lasted between 90 and $110 \mathrm{~min}$. As indicated below, the validation resulted in a sensitivity of $62 \%$, a specificity of $89 \%$ and a kappa $=.80$ for the Tamil version of the UCLA PTSD-Index.

\section{Results}

Responding to the Event List, almost all children reported experiencing or witnessing a series of life threatening events. Only $8 \%$ of the sample reported no traumatic experiences. $79 \%$ reported combat experience, with $58 \%$ having witnessed bombing, $40 \%$ shelling, and $30 \%$ having experienced an attack on their homes. Furthermore, $40 \%$ of the children had witnessed the death of a person. These figures were validated by the independent interview of the clinical experts with one exception: when interviewed by teachers, only $1 \%$ of the children reported having been sexually abused, whereas this figure amounted to $9 \%$ in the validation interview.

These figures are consistent with the independent answers of both parents and children in the UCLA-questionnaire, with 93\% of the children having disclosed traumatizing events. $89 \%$ of the children were horrified and $71 \%$ felt helpless when experiencing these events. $57 \%$ of the children reported that these experiences and the resulting symptoms would interfere with their life through social withdrawal, difficulties leading a normal family life, and problems in school performance. The diagnosis of a PTSD is likely when a traumatic event has elicited sufficient symptoms in three prominent categories, with sufficient intensity to interrupt normal functioning. These criteria were met by $29 \%$ of the children, according to their own self-report, and in $27 \%$ of the cases when questions were answered by the caretaker.

The validation by structured interviews verified these figures in all but one school. The percentage for agreement is as follows for six different schools: $100 \%, 87 \%, 25 \%, 100 \%, 75 \%$, and $83 \%$. The reason for the low correspondence (25\%) in one of the schools was that the teacher's interview resulted in an unlikely $100 \%$ PTSD-prevalence (whereas the figure obtained in the validation was a more typical 25\%). Our investigation of high endorsement of PTSD by the teachers in this school did not 

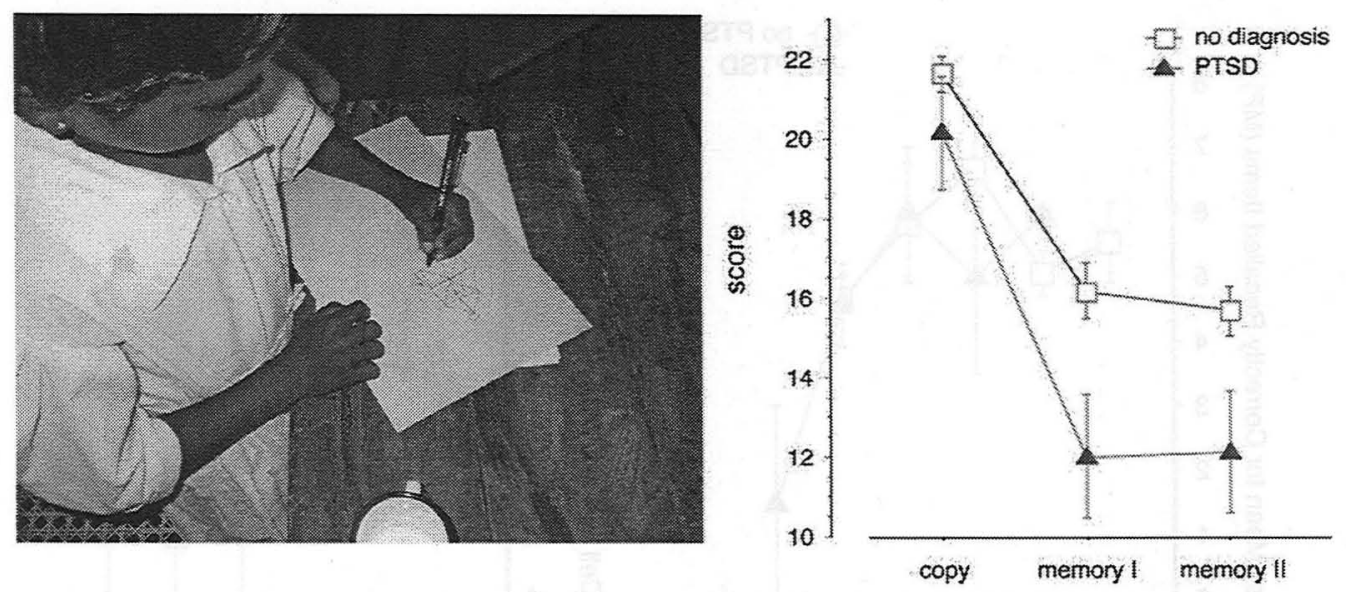

Fig. 1. Left: A child is drawing the Rey-Figure from memory; Right: memory performance in the Rey-Figure test is better in children without PTSD (squares) than in those who present with PTSD (triangles). Error bars indicate standard errors.

reveal a lack of understanding of how to administer the instrument; rather, it seems as if individual reasons in conjunction with political motivation produced a significant bias when completing the forms. We therefore excluded this school from the analysis, leaving $N=350$ for the whole sample and $N=53$ for the validation part. This resulted in a sensitivity of $62 \%$, a specificity of $89 \%$ and a kappa $=.80$ for the Tamil version of the UCLA PTSD-Index and a PTSD rate of 23\% (no PTSD/PTSD, boys: $127 / 37$, girls 144/42). This corresponded to the judgement of the parent/caregiver with $24 \%$ PTSD point prevalence. Kappa $=.77$ for this judgement was somewhat lower, but the correspondence in $79 \%$ of cases, between these two independent assessments of the child's mental health, was high and remarkable given the number of children a parent often had to care for. The PTSD prevalence according to the clinical interviews was $26 \%$ and the life-time prevalence in this sub-sample was $36 \%$. From these data it can be concluded that about one-fourth of the children included in the survey suffer from chronic PTSD.

The neuropsychological testing and the school grades validated the outcomes further and accentuated problems associated with these mental conditions: The Rey-Osterrieth Complex Figure test revealed that children generally copied the figure nearly perfectly. The interaction of PTSD diagnosis with the time of drawing (copy, memory I and memory II) $[F(2,126)=3.1$, $P<.05]$ and a main effect for PTSD $[F(1,63)=6.7, P=.01]$ show that, on average, traumatized children are less able to recall the different elements of the Rey-Figure correctly [see Fig. 1; main effect recall $F(2,126)=98.8$ ]. The recall score (\% correctly recalled elements) drops from $74 \pm 19 \%$ (mean \pm SD) in the children without PTSD to $56 \pm 24 \%$ in those suffering from PTSD $[F(1,63)=9.1, P<.005]$.

The deficit in memory functions is further supported by the MP-test for incidental learning. Children without PTSD diagnosis assigned an average of $5.9 \pm 1.8$ objects correctly, whereas the traumatized children managed to correctly place only $4.5 \pm 1.9$ objects out of the ten $[F(1,57)=7.5, P<.01]$. A quadratic trend $[r=.32 ; F(2,64)=3.7, P<.05 ; t=1.9$ for the linear and $t=2.4, P=.02$ for the quadratic regression coefficient] indicated that his performance was lower for the highest numbers of traumatic event types experienced by the child. Fig. 2 illustrates this relationship, whereby the number of events was divided into six classes. As evident, those children with the greatest load of traumatic stress (class 5) displayed the weakest performance; in this case, class 5 includes only children with PTSD. When a similar grouping of the number of events was related to performance in the Rey-Figure test, a performance difference was observed for class 4 and seems also only present in children with a PTSD diagnosis (Fig. 2, right).

The clinical expert interview further revealed anxiety disorders other than PTSD in $8 \%$ of the children without PTSD ( 2 children with separation anxiety, 1 social phobia, 1 agoraphobia) and co-morbid with PTSD in $12 \%$ of the children ( 1 agoraphobia, 1 panic). Three children (5\%) who met the diagnosis of Attention-Deficit-Hyperactive-Disorder and one child with conduct disorder had no PTSD. 68\% of the traumatized children responded affirmatively to at least one of the M.I.N.I. screening questions related to affective disorders, whereas this was the case in only $12 \%$ in children without PTSD ( $\chi^{2}=19.0$, $P<.001$ ). Correspondingly, $41 \%$ in the PTSD group ( 6 dysthimia, 1 current major depression), but only $4 \%$ in the group without PTSD met a diagnosis for an affective disorder $\left(\chi^{2}=15.1, P<.001\right)$. A current risk for suicide (B2-B6, M.I.N.I.) was diagnosed in $26 \%$ of the children who presented with PTSD ( $7 \%$ in children without PTSD; $\chi^{2}=3.9, P<.05$ ).

In the whole survey sample, somatic symptoms were more frequently reported in children with PTSD ( $4.5 \pm 2.0$ in the last 4 weeks) than in those without PTSD $[3.4 \pm 1.8 ; F(1,313)=13.0, P<.001]$. A corresponding difference was obvious within as well as across schools, for instance, when average differences for each of the 15 schools were examined [ 1.5 more somatic symptoms for PTSD; $t(14)=4.3, P<.001]$. Moreover, the same difference was observed in the validation study [5.50 vs. 3.96 , $F(1,63)=6.3, P<.02$ ]. For instance, $70 \%$ of the children with PTSD reported to have suffered from headaches during the last 4 weeks. This was the case in only $53 \%$ in those without PTSD $\left(\chi^{2}=4.7, P<.05\right.$; headaches is a sign that typically appears in traumatized people.) 

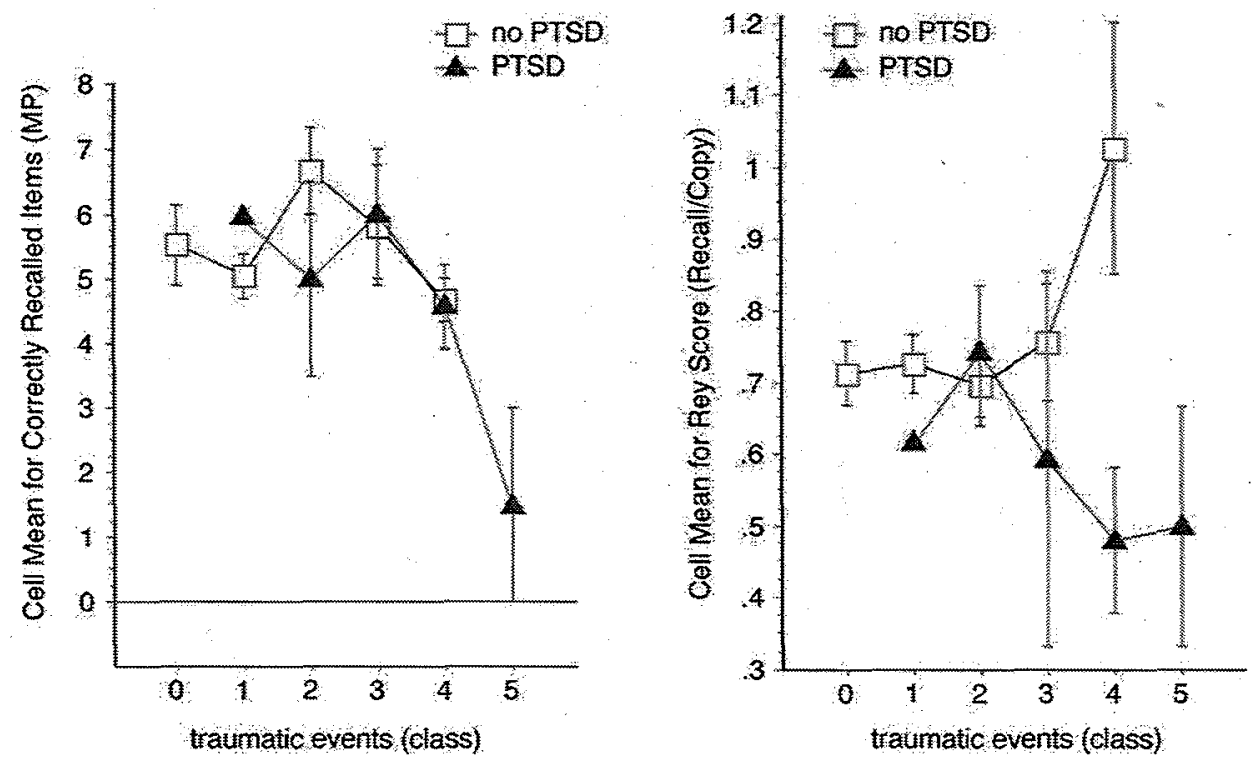

Fig. 2. The number of traumatic event types (witnessed and experienced were pooled) was divided into six classes. There were no traumatic events in class 0 and, therefore, no children with PTSD in this class. In contrast, children with the highest event load (class 5) all presented with PTSD and also showed poor recall (left MP-test, items correctly remembered on the ordinate, right proportion of recall in the Rey-Figure test as a fraction of the performance when copying the figure).

Table 1

Grades of school children with and without a diagnosis of PTSD (grades range from zero to the best possible achievement represented by a score of 99).

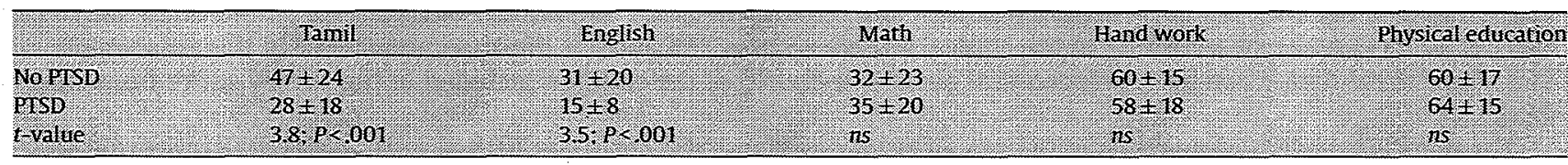

The children's grades in school, when averaged separately for the two groups and across disciplines, reflect that the problems in functioning seem not to appear in physical education and hand work, but focus on deficit in the verbal abilities, possibly a problem relating to communication skills (Table 1, values for the full sample). Examination of the differences within schools confirms this difference and thus exclude the possibility that clustering may have produced the effects. (On average, children without PTSD scored better in all of the schools in Tamil, and in all but one in English grades.)

This result was further supported by the teachers' ratings on how well children comprehend Tamil $(P<.01)$ and English $(P<.01)$. Comparatively, there was no such difference in the rating of overall motoric abilities and in the ability to make use of mathematical skills. This deficit in verbal abilities was present irrespectively of the magnitude of traumatic events a child would report (Fig. 3). The impairment in self-reported functioning, on the other hand, increased with the magnitude of events even in those children without PTSD. In both groups, somatic complaints were higher in those who reported traumatic experiences (Fig. 3).

Children with PTSD had spent more time in internment camps $(2.9 \pm 4.8$ vs. $1.4 \pm 2.5$ years, $P<.01)$ and more frequently reported that family members had died $(.35 \pm .63$ vs. $.10 \pm .32 ; P<.0001)$. There was no significant difference between the children with and without PTSD in terms of parental income, number of bikes, or amount of livestock owned by the family. However, children without PTSD reported that they had an average of $19 \pm 4$ meals per week, compared to $16 \pm 6$ in children with PTSD (Mann-Whitney, $z=2.45, P<.02$ ); there was a corresponding difference in the number of curries with 1.9 vs. $1.6 ; z=2.1, P<.05)$. Children with PTSD, compared to those without, owned fewer clothes $(3.7$ vs. $6.0, P<.001)$, toys $(0.5$ vs. $1.4, P=.001$ ), books ( 3.8 vs. $4.9, P<.01$ ), notebooks ( 3.0 vs. $5.1 P<.001$ ). They also reported that their mothers and fathers owned fewer clothes than their healthy peers did (mother 4.0 vs. $6.3, P<.001$, father 2.7 vs. $4.4, P<.001$ ). In order to test if economic disadvantages might have played an essential role in the development and maintenance of PTSD, a binary logistic regression was calculated with PTSD as the dependent and traumatic event load and the above noted economic indices as independent variables. The event score had the greatest explanatory power (partial $r=.24$ ), measures for the possession of the child (clothes, $r=-.11$, notebooks $r=-.09$ ) but not of the parents $(r=0.0)$ contributed also to atotal variance explained of $r^{2}=.21$

\section{Discussion}

The present study pursued two goals: with a perspective to developing an adequate community-based psychosocial program, the ability and reliability of trained teachers for epidemiological and diagnostic assessments was evaluated. Second, 

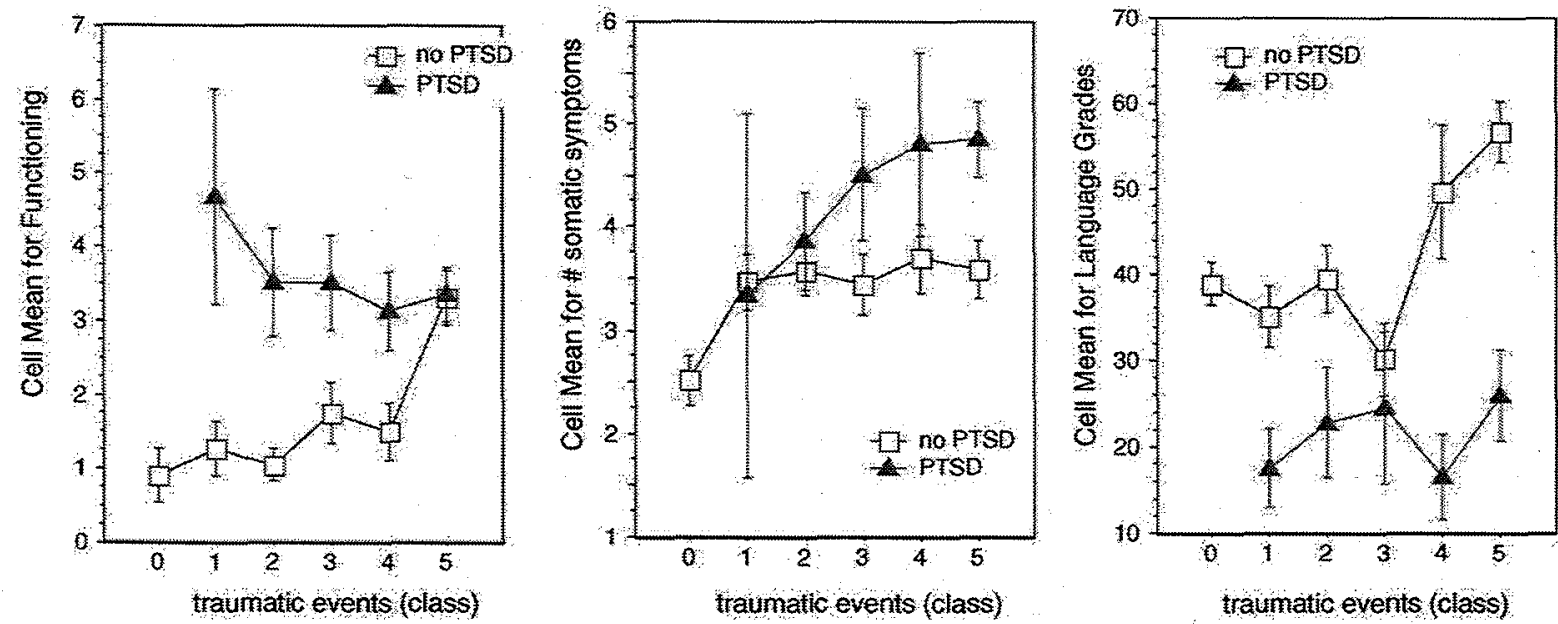

Fig. 3. The abscissa represents the number of different traumatic events experienced, divided into six classes. The different graphs show (a) the impairment in self-reported functioning (left), (b) the number of somatic symptoms during the last months and (c) the average grade in Tamil and English (reading and dictation, the grading system ranges from 0 to 100 , with 100 being the best grade). These three variables are graphed separately for the children with and without PTSD. In contrast to the data from the validation study presented in Fig. 2 , these data include the full sample.

the impact of repeated traumatic stress, in this case caused by the long-lasting civil war on mental health and functioning of children was to be examined.

The epidemiological study provided an exact and quantitative answer to the question of how well teachers can perform screening after a short and focused training. In five of the six schools included in the survey, teachers achieved valid results, as confirmed by the corresponding outcome of the expert screening. In a sixth school, the 100\% PTSD-prevalence outcome was not valid. Post hoc inquiry did not indicate that the corresponding teachers did not understand the application of the instrument; rather, it seemed that political motivation had produced a significant bias.

Thus, the present study demonstrates that standardized diagnostic screening instruments can be adapted to local cultures in resource-poor countries and can be reliably applied by trained local psychosocial workers or teachers. It seems noteworthy, however, that all teachers reported to have experienced traumatic stress repeatedly. This may explain in part why teachers were quick in grasping the concept and highly motivated to perform the interviews.

The study revealed a high number of traumatizing experiences in children, and it is therefore not surprising that about one in four children suffers from PTSD. Adverse consequences of the traumatic experiences on mental health and normal development were disclosed: Both the memory tests and the school grades demonstrated significant impairment of cognitive development. Within the group of children with PTSD, memory performance was even lower in those who reported a greater variety of traumatic experiences. Beers and DeBellis (2002) tested children with maltreatment-related PTSD and showed, in agreement with the present findings, that children with PTSD completed a poorer recalled copy of the Rey-Osterrieth Figure. Previous findings, however, included a smaller number of subjects and no comparison with children who had been exposed to traumatic stress, but did not develop PTSD. The present findings show how both factors interact: Traumatic stress alone, even in the classes with the high event loads (Fig. 2), is not sufficient to reduce memory performance. However, when PTSD occurs, test performance is worse in those children who report higher event loads. Part of the memory testing ("memory II" in Fig. 1) was performed after the clinical interview. This raises the possibility of biasing interference effects on the memory performance from carrying out a PTSD interview in the intervening period. However, the deficits were as marked already prior to the interview ("memory I" in Fig. 1).

In addition to reduced memory performance, traumatized children perform less well in language skills(Tamil and English), but not in math and physical abilities. A high load of traumatic events seems to reduce the ability to function, but the particular type of impairment depended on both the diagnosis and the magnitude of events experienced. Beers and DeBellis (2002) found that children with PTSD performed more poorly on the California Verbal Learning Test in a long delay free recall. A corresponding deficit in our sample of affected children might explain the below average grades in both languages. While alternative interpretations are possible, the selective pattern of grade deficits (Table 1) seems noteworthy, suggesting that PTSD does not simply act through unspecific mechanisms such as attention, which theoretically should also affect grades in math and handwork. The results fit with recent findings from imaging magnetic brain activity in survivors of severe traumatic stress, where spontaneous abnormalities were most pronounced in the left-hemispheric insula (Kolassa et al., 2007) and in Broca's region (Ray et al., 2006).

In children without PTSD, school achievement is better in those who suffer from PTSD, while functioning impairment is greater. However, the sample with the highest class of traumatic experiences demonstrates impairment irrespective of whether or not the PTSD criteria are fulfilled. In addition, children with PTSD also presented with more somatic complaints (Fig. 3). Despite the fact that the data are correlational in nature, we argue that alterations in memory functions and somatic consequences associated with PTSD increase the load and burden to such an extent that school performance becomes 
impaired. The reverse causality, i.e., that poor school performance degrades memory and makes PTSD more likely, seems difficult to reconcile with the data, as it would not explain why children with a PTSD diagnosis perform worse in Tamil and English, but do not show reduced performance in math, science, handwork or sports. Neither can PTSD alone explain the variation of the group differences with traumatic event load.

The failure of PTSD children in domains where language is important may thus be explained as consequence of impaired memory function. In addition, neuropsychological testing suggests that the spatial memory required to weave sensory experiences in a context is also weakened with cumulating traumatic experience. Both of these functions are essential to build autobiographic memory, so that the present findings are consistent with models of PTSD that involve a contextual memory deficit at its core. They are also consistent with the notion that the creation of verbal access to the traumatic memories may have a beneficial effect (Neuner, Schauer, Klaschik, Karunakara, \& Elbert, 2004b; Schauer, Neuner, \& Elbert, 2005). Currently, our knowledge about effective and efficient treatment possibilities in children is insufficient. The magnitude of the observed effects call for scientific investigations to allow treatment of affected children in conflict zones.

From a humanitarian perspective, community-based awareness of the consequences of traumatic stress, both as a preventative measure and as a way of decreasing stigmatization of affected individuals should be encouraged. It is important to keep in mind that trauma experienced by children in conflict regions is interrelated with contextual political and social factors. Individual symptoms as a result of wartime trauma indicate first and foremost a "disturbed society" (NCTSN, 2003), even though the mental, emotional and physical disturbance is detected in individuals. Parents, caretakers, teachers, peers and other relevant members of the child's (post-) war community need to be informed and educated about psycho-social problems, which can occur in children as well as those who care for them, given the high stress context. Knowledge about consequences of traumatic stress, validation of problems e.g. such as drop in school grades due to concentration issues, empathy and positive appraisal by caretakers might help mediate symptoms. Community information on access to mental health services and psycho-social support for the child as well as the family may reduce helplessness and aggravation of suffering.

\section{Acknowledgements}

We greatly acknowledge the advice of Professor Daya Somasundaram, Mr. Anthony Anandarajah and the GTZ staff, the work of the Tamil/English translators, and comments of Professor Brigitte Rockstroh on the manuscript. We thank all the children and teachers who supported our work with great enthusiasm, trust and openness.

\section{References}

Almqvist, K., \& Brandell-Forsberg, M. (1997). Refugee children in Sweden: Post-traumatic stress disorder in Iranian preschool children exposed to organized violence. Child Abuse \& Neglect, 21, 351-366.

Arroyo, W., \& Eth, S. (1985). Children traumatized by Central American warfare. In S. Eth \& R. S. Pynoos (Eds.), Children (pp. 101-120). Washington, DC: American Psychiatric Press.

Beers, S. E., \& DeBellis, M. D. (2002). Neuropsychological function in children with maltreatment-related posttraumatic stress disorder. American journal of Psychiatry, 159, 483-548.

Bracken, B. A. \& Barona, A. (1991). State of the art procedures for translating, validating, and using psychoeducational tests in cross-cultural assessment. Psychology International, 12, 119-132.

Charmandari, E., Kino, T., Souvatzoglou, E., \& Chrousos, G. P. (2003). Pediatric stress: Hormonal mediators and human development. Hormone Research, $59(4), 161-179$

DeQuervain, D., Kolassa, I., Ertl, V.; Onyut, P. L, Neuner, F., Elbert. T., Papassotiropoulos, A. (2007). A deletion variant of the a2b-adrenoceptor is related to emotional memory in Europeans and Africans. Nature Neuroscience. July 29; [Epub ahead of print].

Dyregrov, A., Gupta, L., Gjestad, R., \& Mukanoheli, E. (2000). Trauma exposure and psychological reactions to genocide among Rwandan children. Journal of Traumatic Stress, 13, 3-21.

Elbert T.. Huschka B., Schauer E., Schauer M.(2003). vivo evaluation report on the psychosocial-counselling component of GIZ Vanni Education Rehabilitation Project. Available at <http://vivo.org> Accessed December 2006.

Elbert, T., Rockstroh, B., Kolassa, I. T., Schauer, M., \& Neuner, F. (2006). The Influence of organized violence and terror on brain and mind-A co-constructive perspective. In P. Baltes, P. Reuter-Lorenz, \& F. Rösler (Eds.), Lifespan development and the brain: The perspective of biocultural co-constructivism (pp. 326-349). Cambridge: Cambridge University Press.

Karunakara, U., Neuner, F., Schauer, M., Singh, K. Hill, K., Elbert, T., \& Burnham, G. (2004). Traumatic events and symptoms of post-traumatic stress disorder amongst Sudanese nationals, refugees and Ugandan nationals in the West Nile. African Health Sciences, 4, 83-93.

Kim, J. J., \& Diamond, D. M. (2002). The stressed hippocampus, synaptic plasticity and lost memories. Nature Reviews Neuroscience, 3, $453-462$.

Kinzie, J. D., Sack, W., Angell, R. Clarke, G., \& Ben, R. (1989). A three-year follow-up of Cambodian young people traumatized as children. American Academy of Child Adolescence Psychiatry, 28, 501-504.

Kolassa, I. T., Wienbruch. C., Neuner, F., Schater, M., Ruf, M., Odenwald, M., \& Elbert, T. (2007). Altered oscillatory brain dynamics after repeated traumatic stress. BMC Psychiatry, 7, 56.

Lezak, M. D. (1995). Neuropsychological assessment. New York: Oxford University Press.

McEwen, B. (2002). The end of stress as we know it. Joseph Henry Press/Dana Press.

NCTSN. (2003). Review of child and adolescent refugee mental health. Boston. USA: National Child Traumatic Stress Network.

Neuner, F., Schauer, M., Karunakara, U., Klaschik, C., Robert, C., \& Elbert, T. (2004). Psychological trauma and evidence for enhanced vulnerability for PTSD through previous trauma in West Nile refugees. BMC Psychiatry, 4, 34

Neuner, F., Schauer, M., Klaschik, C., Karunakara, U., \& Elbert, T. (2004). A comparison of narrative exposure therapy, supportive counselling, and psychoeducation for treating posttraumatic stress disorder in an African refugee settlement. Journal of Consulting and Clinical Psychology. 72, 579-587.

Neuner, F., Schauer, E., Catani, C., Ruf, M., \& Elbert, T. (2006). Post-Tsunami stress: A study of posttraumatic stress disorder in children living in three severely affected regions in Sri Lanka. Journal of Traumatic Stress, 19, 339-347.

Ray, W. J., Odenwald, M., Neuner, F., Schauer, M., Ruf, M., Rockstroh, B., \& Elbert, T. (2006). Decoupling neural networks from reality: Dissociative experiences in torture victims are reflected in abnormal brain waves in left frontal cortex. Psychological Science, 17, 825-829. 
Realmuto, G. M., Masten, A., Flies, L., Hubbard, J., Groteluschen, A., \& Chun, B. (1991). Adolescent survivors of massive childhood trauma in Cambodia: Life events and current symptoms. Journal of Traumatic Stress, 5, 589-599.

Saigh, P. A. (1991). The development of posttraumatic stress disorder following four different types of traumatization. Behavioural Research and Therapy. 29. 213-216.

Sapolsky, R. M. (1992). Stress, the aging brain, and the mechanisms of neuron death. Boston: MIT Press.

Schaal, S., \& Elbert, T. (2006). Ten years after the genocide: Trauma confrontation and post-traumatic stress in Rwandan adolescents. Journal of Traumatic Stress, 19, 1-11.

Schauer, M., Neuner, F., \& Elbert, T. (2005). Narrative Exposure Therapy. A short-term intervention for traumatic stress disorder after war, terror or torture. Göttingen: Hogrefe \& Huber.

Sheehan, D. V., Lecrubier, Y., Sheehan, K. H., Amorim, P., Janavs, J., Weiller, E., Hergueta, T., Baker, R., \& Dunbar, G. C. (1998). The Mini-International Neuropsychiatric Interview (M.I.N.I.): The development and validation of a structured diagnostic psychiatric interview for DSM-IV and ICD-10. Journal of Clinical Psychiatry, 59(Suppl. 20), 22-33.

Smith, P. Perrin, S., Yule, W., Hacam, B., \& Stuvland, R (2002). War exposure among children from Bosnia-Hercegovina: Psychological adjustment in a community sample. Journal of Traumatic Stress, 15, 147-156.

Somasundaram, D. (2002). Child soldiers: Understanding the context. British Medical Journal, 324, 1268-1271.

Steinberg, A. M.. Brymer, M. J., Decker, K. B., \& Pynoos, R. S. (2004). The university of California at Los Angeles post-traumatic stress disorder reaction index. Current Psychiatry Reports, 6, 96-100.

Thabet, A. A., \& Vostanis, P. (2000). Post-traumatic stress disorder reactions in children of war: A longitudinal study. Child Abuse $\&$ Neglect, $24,291-298$.

Yule, W., \& Canterbury, R. (1994). The treatment of post-traumatic stress disorder in children and adolescents. International Review of Psychiatry, 6, $141-150$. 